\title{
COMPUTATION E INFORMATICA
}

Recibido 23 Jun 2021

ReCIBE, Año 10 No. 1, Mayo 2021

Aceptado 08 Sep 2021

\section{Dimensión epistemológica aplicada a procesos de social media de un grupo académico de facebook}

\section{Epistemological dimension applied to social media processes of a facebook academic group}

Paola E. Velazquez Solis ${ }^{1}$

paola.velazquez@uabc.edu.mx

Brenda L. Flores-Rios ${ }^{1}$

brenda.flores@uabc.edu.mx

${ }^{1}$ Instituto de Ingeniería, Universidad Autónoma de Baja California,Mexicali, Baja California, México. 


\title{
RESUMEN
}

Las instituciones educativas están incorporando procesos de social media en sus estrategias, como una fuente de conocimiento a partir de la socialización entre los diversos roles. Es por ello que se buscan metodologías, como las empleadas en la Gestión de Conocimiento, que apoyen la integración de nuevos procesos a la dinámica de sus necesidades. En este documento, se emplea la metodología KoFI para identificar los elementos involucrados en la dimensión epistemológica y establecer una correspondencia entre los flujos de conocimiento con los procesos de social media y las herramientas tecnológicas que se utilizan para descubrir, recopilar, preparar y analizar los datos. Se concluye que la implementación de la Gestión de Conocimiento proporciona un complemento a la ejecución de las actividades de los procesos de social media, realizando una contribución teórica-práctica para la adopción de las redes sociales en la comunicación de un grupo académico. Así como analizar los contenidos compartidos y las publicaciones de Facebook en función al engagement.

\section{PALABRAS CLAVE}

Flujos de conocimiento, Dimensión epistemológica, Metodología KoFI, Procesos de social media, Redes sociales.

\begin{abstract}
Educational institutions are incorporating social media processes in their strategies, as a source of knowledge from the socialization between the various roles. It is because of that methodologies are sought, such as those used in Knowledge Management, which supports the integration of new processes to the dynamics of their needs. In this document, the KoFI methodology is used to identify the elements involved in the epistemological dimension and establish a correspondence between the knowledge flows with the social media processes and the technological tools used to discover, collect, prepare and analyze data. It is concluded that the implementation of Knowledge Management provides a complement to execution of the activities of the social media processes, making a theoretical-practical contribution to the adoption of social networks in the communication of an academic group. As well as analyzing shared content and Facebook posts based on engagement.
\end{abstract}

\section{KEYWORDS}

Knowledge flows, Epistemological dimension, KoFI Methodology, Social media processes, Social networks. 


\section{INTRODUCCIÓN}

El conocimiento es un recurso importante para que las organizaciones aumenten el aprendizaje organizacional, resuelvan problemas y desarrollen nuevos procesos desde el nivel individual hasta el interorganizacional (Dimensión ontológica) (Nisar et al., 2018). Así mismo, el conocimiento es un flujo en el que se mezclan las experiencias, valores, información contextual y puntos de vista de expertos que facilitan un marco de análisis para evaluar e incorporar nuevas experiencias e información (Flores-Rios, 2016). Los procesos organizacionales utilizan diversos canales de comunicación, tanto formales como informales, para almacenar y gestionar el conocimiento (Nisar et al., 2018). Según lo propuesto por diversos autores (Alavi \& Leidner, 2003; Flores-Rios, 2016; Nonaka \& Takeuchi, 1995), la utilidad de especificar diferentes tipos de conocimiento radica en comparar las ventajas que se derivan de cada uno de ellos. En la dimensión epistemológica (Nonaka \& Takeuchi, 1995), se utilizan dos tipos de conocimiento: tácito y explícito. El conocimiento tácito es más complejo de articular debido a que surge por parte de la experiencia con relativa facilidad de una persona a otra (Flores-Rios, 2016). A diferencia del conocimiento explícito el cual se refiere al conocimiento representado por símbolos, números, palabras, fórmulas y es transferido para generar más conocimiento explícito o comunicado en un lenguaje para generar nuevo conocimiento tácito (Cantú Ballesteros et al., 2014; Flores-Rios et al., 2014; Nonaka \& Takeuchi, 1995; Rodriguez-Elias \& Martínez G., 2011). La Gestión de Conocimiento (GC) promueve un enfoque integrado para identificar, capturar, compartir, recuperar y evaluar todos los activos intangibles de las organizaciones, incluido el conocimiento explícito existente en las bases de datos, documentos y procedimientos (Nisar et al., 2018), así como el conocimiento tácito. Por lo tanto, el flujo de conocimiento se define como el conocimiento que es transferido de una persona o lugar, a otro, donde la transferencia del saber cómo, consiste en la experiencia que tiene valor estratégico para una organización (Erden et al., 2014; Flores-Rios, 2016; Giraldo Marín et al., 2021).

Una buena estrategia de GC consiste en orientar los esfuerzos de análisis de flujos de conocimiento a los aspectos de mayor impacto en la organización (Flores-Rios, 2016; Perez-Soltero et al., 2007) considerando los procesos de conocimiento para la aplicación, transferencia y evolución del conocimiento en la generación o innovación de productos y servicios (Claver-Cortes et al., 2008; Rodriguez-Elias \& Martínez G., 2011). Esta nueva concepción identifica la necesidad de las organizaciones de cómo implementar estrategias, técnicas o herramientas de GC en las necesidades de conocimiento que se les presentan en las redes sociales (Narazaki et al., 2020). Un caso particular es el de los docentes o académicos de cualquier disciplina o cultura, están comenzando a impartir cursos a través de plataformas como redes sociales apoyados con las diferentes funcionalidades que brindan las herramientas disponibles, como Portal Home, Historias de voz y el almacenamiento en la nube de Facebook, con lo que se incentiva a los estudiantes a realizar una participación más activa. Propiciando la modificación de las prácticas tradicionales a los contenidos temáticos de los programas de enseñanza-aprendizaje en las instituciones educativas (Velazquez-Solis et al., 2021). La transparencia y acceso al contenido que se presenta en las redes sociales elimina la limitación de la GC, al tratar el conocimiento como un insumo, debido a que las contribuciones o publicaciones están disponibles para todos los usuarios que posean permisos de acceso a ellas. Cuando una herramienta tecnológica registra automáticamente cómo se realiza la interacción en plataformas digitales, captura el conocimiento tal como está integrado en la práctica, en lugar de preservarlo como una abstracción (Kane, 2017). Se ha observado que existe una relación entre los procesos de creación y la GC en ambientes de redes sociales y las características intrínsecas de los procesos de social media (Helms et al., 2017). Sin embargo, se considera de relevancia identificar cuáles otros tipos y procesos de conocimiento se asocian con los procesos de social media, partiendo de la diferencia entre las redes sociales y los social media. 
Los social media se refiere a los espacios virtuales donde los usuarios comparten intereses, información, experiencias, material audiovisual, se incentiva la participación entre el comunicador y la audiencia, con el propósito de generar una conversación multidireccional, generar proyectos, integrar comunidades y diseñar nuevas formas de entretenimiento y acción social colectiva (Ureña et al., 2019); convirtiéndose en plataformas de cambio social (van Rooyen, 2020). Por otro lado, las redes sociales como Facebook, Instagram, Twitter, WhatsApp, son medios en los cuales las organizaciones recurren para comunicarse con su personal o como sociedad (Vranešević et al., 2019), caracterizadas por tener una base de usuarios amplia y heterogénea con participación intermitente. Se ha generado un crecimiento de servicios de datos y la necesidad de aprovechar el uso de técnicas y herramientas de social media en procesos educativos. Esta afirmación se basa en los resultados de un mapeo sistemático de literatura para la identificación de áreas de aplicación de arquitecturas de software basadas en modelos, técnicas y herramientas de social media (Velazquez-Solis et al., 2021) donde además, se identificaron las siguientes problemáticas en el análisis de flujos de conocimiento: 1) la interacción de los usuarios en las redes sociales requiere de un alto nivel de compromiso (Ballesteros Herencia, 2018) para obtener datos que realmente aporten valor en el análisis de los mismos; 2) los resultados estadísticos varían dependiendo de la cantidad de usuarios y sus interacciones; 3 ) la interpretación de un reporte generado por el análisis de datos requiere de una capacitación técnica por parte del arquitecto de software; y 4) la necesidad de documentar las experiencias del análisis de las interacciones de los usuarios en las redes sociales en función de los flujos de conocimiento y contexto del analista de redes sociales.

Por otro lado, se señala que existe una necesidad de recopilar información de la participación de los usuarios, considerando no solo la transparencia en los datos, sino también el riesgo en la predicción de sentimientos y el análisis de reacciones, emociones y opiniones (Mushtaq et al., 2018). A partir de la revisión de trabajos enfocados en Facebook (Arora et al., 2019; Ballesteros Herencia, 2018; Bazarova et al., 2015; Hu \& Chen, 2016; Peruta \& Shields, 2017) y el análisis de los contenidos en las publicaciones de Facebook, se identificó la medición del engagement en los medios digitales, como un valor cuantitativo de la interacción bidireccional entre las organizaciones y los usuarios de redes sociales (Oviedo-García, Muñoz y Castellanos, 2014). Por ello, conocer el engagement a través de la conducta mostrada por los usuarios mediante su actividad en las plataformas digitales, se obtienen con una serie de métricas, cuantificando variables como el número de visitas, el tiempo de permanencia, y el número de seguidores o clicks del tipo "Me gusta", el compartir las publicaciones y los comentarios. Por lo anterior, la aportación de este trabajo es identificar los elementos involucrados dentro de la dimensión epistemológica, estableciendo una correspondencia entre los flujos de conocimiento con los procesos de social media y la identificación de herramientas tecnológicas que les brinden soporte. Así como, el engagement de los miembros de un grupo académico privado de Facebook.

Este documento está estructurado de la siguiente manera: en la sección 2 se describe un marco referencial para la conceptualización de dimensión epistemológica y los procesos de social media; en la sección 3 se presenta la descripción de la metodología KoFI implementada; la sección 4 expone los resultados obtenidos por medio de la especificación y modelado de los procesos de social media bajo un enfoque de flujos de conocimiento aplicado a un estudio de caso. Finalmente, la sección 5 presenta las discusiones y conclusiones del trabajo. 


\section{MARCO REFERENCIAL}

\section{Dimensión epistemológica}

La creación de conocimiento organizacional se representa por medio de dos dimensiones: epistemológica y ontológica (Nonaka \& Takeuchi, 1995). Específicamente, en la dimensión epistemológica el proceso de creación de conocimiento es un proceso dinámico e interactivo, donde el modelo SECI (por las iniciales de cuatro formas de conversión del conocimiento: Socialización, Exteriorización, Combinación e Interiorización) tiene como objetivo conocer cómo se genera nuevo conocimiento y cómo se relacionan entre sí (Palacios Ospina, 2017) dentro de la espiral de conocimiento a través de las cuatro formas de conversión del conocimiento (Nonaka \& Takeuchi, 1995). Se distingue la conversión del conocimiento tácito a tácito (Socialización), de conocimiento tácito a explicito (Exteriorización), de conocimiento explícito a explícito (Combinación) y de conocimiento explícito a tácito (Interiorización) (Diaz Ayon, 2019; Nonaka \& Takeuchi, 1995). La Figura 1 visualiza el modelo SECI identificando los cuatro diferentes patrones de interacción, en los cuales el conocimiento existente es convertido en un nuevo conocimiento tácito y/o explícito (Nonaka \& Takeuchi, 1995).

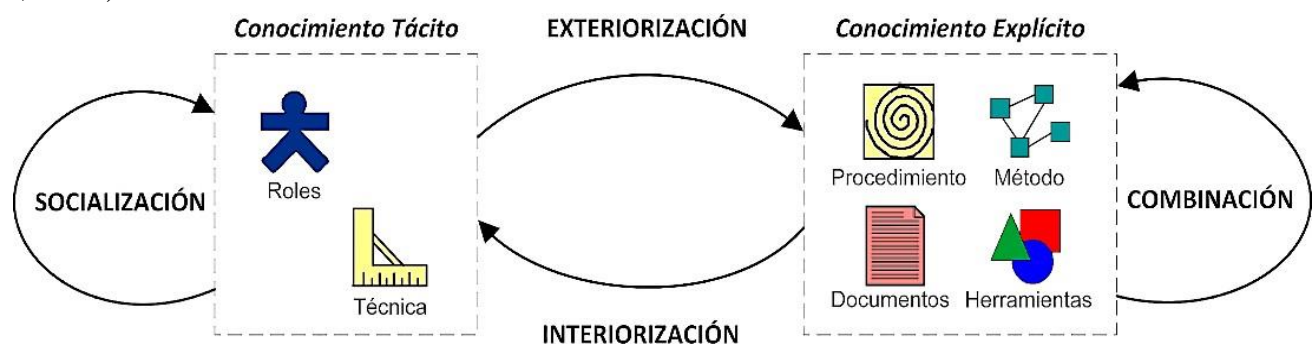

Figura 1. Modelo de procesos de conocimiento. Adaptado de: (Alavi \& Leidner, 2003; Flores-Rios, 2016; Nonaka \& Takeuchi, 1995)

Actualmente, los social media brindan un conjunto de herramientas tecnológicas para la colaboración de equipos de trabajo y el intercambio de conocimiento (procesos de Exteriorización e Interiorización) para una comunicación e interacción fluida entre los miembros de la organización (Socialización) y representan un depósito de conocimiento de código abierto (Ali et al., 2020) necesario en el proceso de Combinación. Esto último, relacionado con otros procesos de conocimiento como el de organización, almacenamiento, codificación y recuperación (Flores-Rios, 2016). Como trabajo relacionado en aplicaciones educativas, se observó cómo el estudio de Díaz Ayon, a partir del modelo SECI identificó la relación de las fases del modelo en el desarrollo de nuevos proyectos o mejora de los servicios en el área de tecnología de las universidades privadas de Lima, Perú (Diaz Ayon, 2019). Del mismo modo, Mora García et al., analizó cómo se gestiona el conocimiento en las universidades públicas Colombianas, y de esta manera identificar los elementos que dan el sustento necesario para proponer estrategias que permitan crear un modelo de GC (Mora Garcia \& De la Cruz Chima, 2018). Giraldo Marin et al., buscaron en un contexto institucional aplicar una metodología que guía estratégica y sistemáticamente el desarrollo de procesos de visibilidad nacional e internacional de la Universidad de Medellín (Giraldo Marín et al., 2021). 


\section{Procesos de social media y las redes sociales}

La tecnología de los social media es una combinación de diferentes recursos que incluyen las redes sociales, wikis, (micro) blogs, marcadores sociales, plataformas de contenido, entre otros, los cuales poseen características distintivas que apoyan los diversos procesos de conocimiento (Helms et al., 2017). La diferencia entre un social media y una red social es que la primera se enfoca en el uso de tecnologías para hacer interactivo el diálogo entre personas; mientras que una red social es una estructura social formada por personas que comparten de manera virtual sus intereses similares (Alawamleh \& Murthy, 2020). Tanto los social media, las redes sociales y los espacios virtuales son mecanismos de interacción (fan page, grupos cerrados, abiertos, públicos, privados, blogs, páginas personales), canales de comunicación con un alcance y potencial de impacto mucho mayor que cualquiera de los otros medios tradicionales, y con la capacidad de interacción y dinamismo superior a cualquiera de los otros medios de comunicación habituales (Briciu \& Briciu, 2021).

El análisis de datos por social media es una herramienta para extraer conocimiento originado en las redes sociales (Helms et al., 2017). El conocimiento obtenido del análisis de estos datos proporciona un conocimiento vivenciado a partir de diversos tipos de interacción, reacción y relación entre varias personas en las redes sociales (Chakraborty et al., 2018). Existen cuatro procesos básicos de social media (Figura 2): el descubrimiento de los datos (Mushtaq et al., 2018), la recopilación, la preparación y el análisis de datos (Goyal \& Goyal, 2020; Gupta \& Gupta, 2016). Los autores del trabajo relacionado resaltan la importancia de determinar el dominio de conocimiento y de dónde se obtienen los datos para su análisis, debido a que la mayor parte de los desafíos por parte de los investigadores aún se encuentran durante este proceso (Liu et al., 2016; Meng et al., 2020). La Figura 2 presenta las actividades asociadas a cada uno de los procesos.

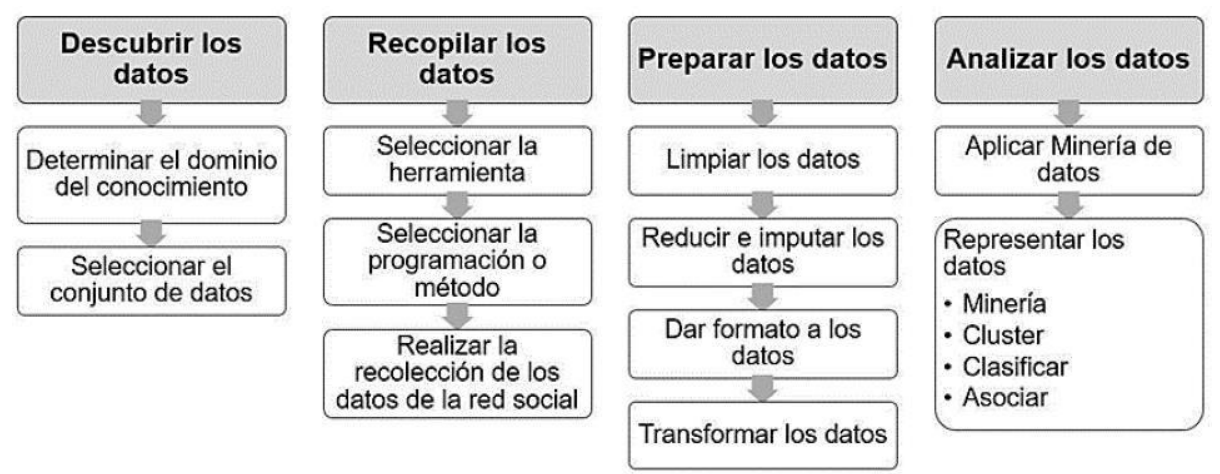

Figura 2. Procesos de social media con sus correspondientes actividades. Adaptado de: (Goyal \& Goyal, 2020; Gupta \& Gupta, 2016; Meng et al., 2020; Mushtaq et al., 2018) 


\section{METODOLOGÍA}

La metodología KoFI (por sus siglas de Knowledge Flow Identification), propuesta por Rodríguez-Elias (2007), define los lineamientos para la identificación y análisis de flujos de conocimiento en los procesos organizacionales, bajo un proceso iterativo e incremental que se puede retroalimentar de una fase a cualquier otra del modelo. Se compone por cuatro fases (Flores-Rios, 2016; Giraldo Marín et al., 2021; Rodriguez-Elias \& Martínez G., 2011): 1) especificar el proceso a analizar y los roles que participan en el proceso; 2) modelar el proceso con enfoque en flujos de conocimiento; 3) analizar el flujo de conocimiento; y 4) analizar las herramientas de soporte al flujo de conocimiento.

La Figura 3 muestra a detalle como la fase 1 define el proceso a analizar, las actividades, los roles y herramientas involucrados, los procedimientos que se utilizan y los documentos o resultados obtenidos en la ejecución de las actividades. En la fase 2, se modela el conocimiento requerido y generado durante las actividades del proceso. La fase 3 permite distinguir las fuentes y los tipos de conocimiento, flujos de conocimiento y las problemáticas en el flujo. Por último, en la fase 4 se realiza un análisis de las herramientas que apoyen los procesos de conocimiento para integrarlas dentro de la eficiencia o automatización del flujo de conocimiento (Cantú Ballesteros et al., 2014; Flores-Rios, 2016; Giraldo Marín et al., 2021; Rodríguez-Elias, 2007) o brindando solución a las necesidades de conocimiento.

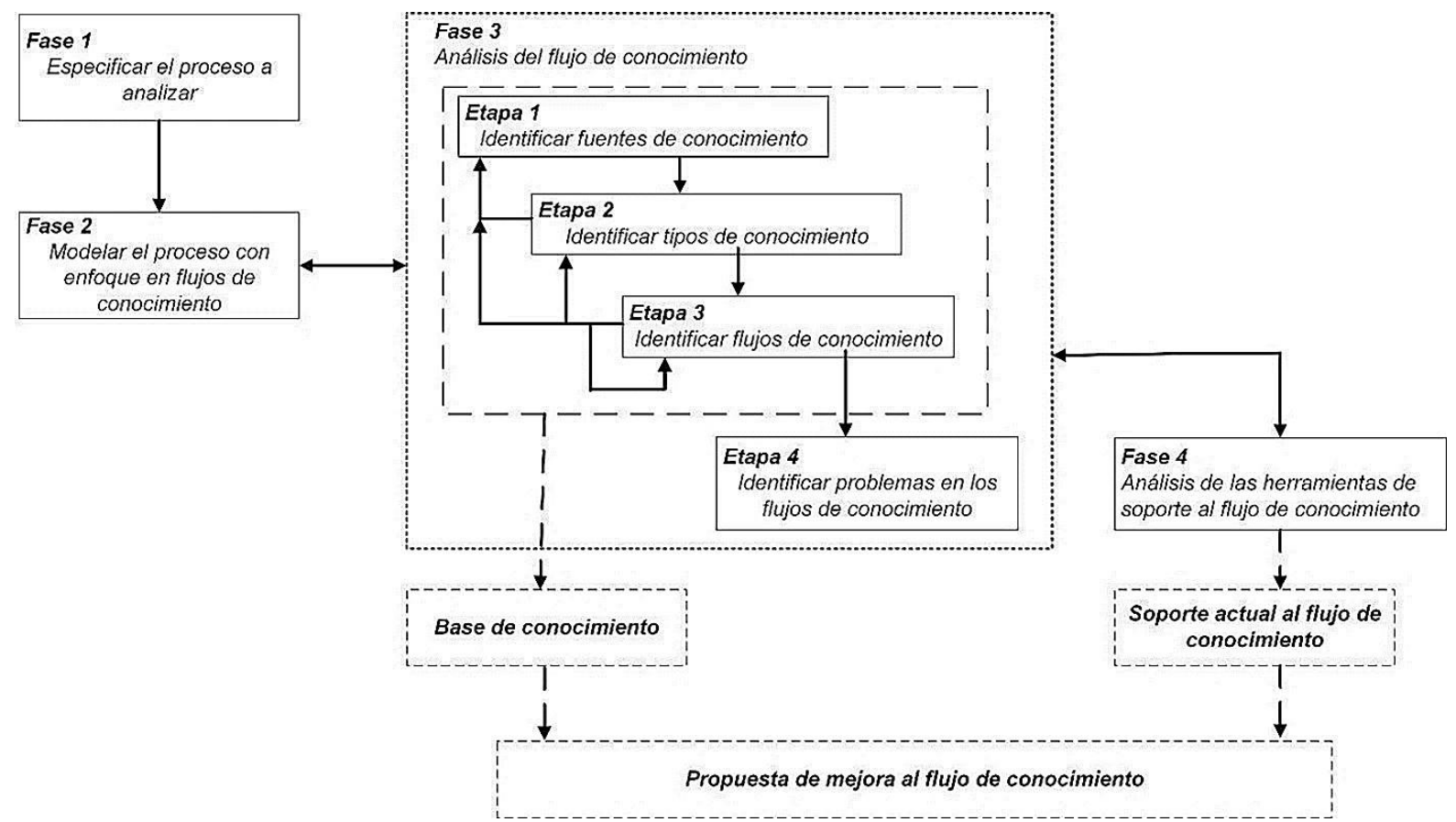

Figura 3. Metodología KoFI. Adaptado de: Flores-Rios (2016)

Para esta investigación, se tomó como estudio de caso un grupo cerrado (privado) de Facebook del programa educativo relacionado con Tecnologías de Información de la Universidad Autónoma de Baja California (GFIC) creado en enero de 2012. El propósito del GFIC es brindar información relacionada a las actividades de los procesos académicos y anuncios, dirigido a 882 miembros conformados por: estudiantes vigentes y no vigentes, egresados y docentes pertenecientes del programa educativo y grupos de interés. 


\section{IMPLEMENTACIÓN}

\section{Fase 1. Especificar el proceso a analizar y roles}

En esta fase se detectaron los roles involucrados en la aplicación de los procesos de social media. La caracterización de los roles, el proceso y el conocimiento son parte de la actividad de determinar el dominio de conocimiento del proceso de descubrir los datos (Figura 2). En la Figura 4 se visualizan los roles obtenidos: el Analista de redes sociales, el Social Media Community Manager, el Coordinador de redes sociales y el Director de redes sociales. Una de las principales tareas de estos roles es que apoyen en mejorar la participación de la comunidad, interactúen con los usuarios (fans, miembros, seguidores), desarrollen una presencia en las plataformas de redes sociales seleccionadas y fomenten comunidades externas. Así mismo, generen un mayor compromiso e iniciativas de alcance para involucrar a la comunidad de manera activa y receptiva. El resultado de esta fase es la selección del conjunto de datos para el periodo de enero a junio 2021, como un reporte semestral de interacciones dentro de la red social Facebook y la especificación del conocimiento tácito y explícito representada bajo el ícono de corchetes (Figura 4).

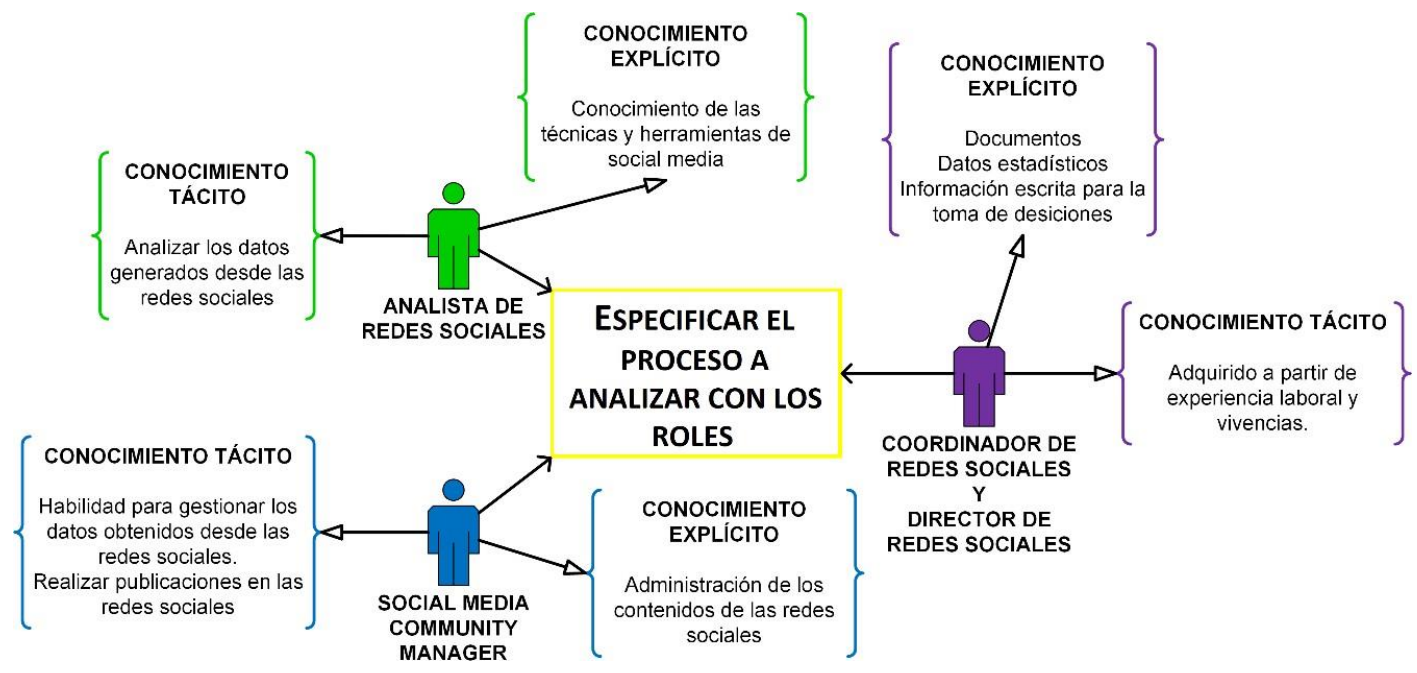

Figura 4. Modelado de roles y tipos de conocimiento identificados

\section{Fase 2. Modelar el proceso con enfoque en flujos de conocimiento}

Para identificar las fuentes de conocimiento del proceso se utilizó el modelado global del ciclo de conocimiento propuesto por (Choo, 1999) por medio de la simbología de gráfica rica adaptada (Flores-Rios, 2016), el cual vincula la teoría de la organización, las Ciencias de la información y las cognoscitivas, con los propósitos de la GC. De esta manera, en la Figura 5 se presenta la identificación de los elementos de percepción, la cual comprende la red social Facebook con las estadísticas obtenidas de las interacciones de los usuarios (API de Facebook), las herramientas de hojas de cálculo, análisis estadístico de datos (SPSS, Anaconda Python, RStudio) (Velazquez-Solis et al., 2021), los procesos para el análisis de datos (Figura 2) y los cuatro roles obtenidos de la fase anterior. Esta fase permitió recopilar los datos del GFIC en la red social Facebook y seleccionar las herramientas tecnológicas requeridas (Hojas de cálculo, SPSS, RStudio y Anaconda Python) y el método de social media (Analizar contenido). 


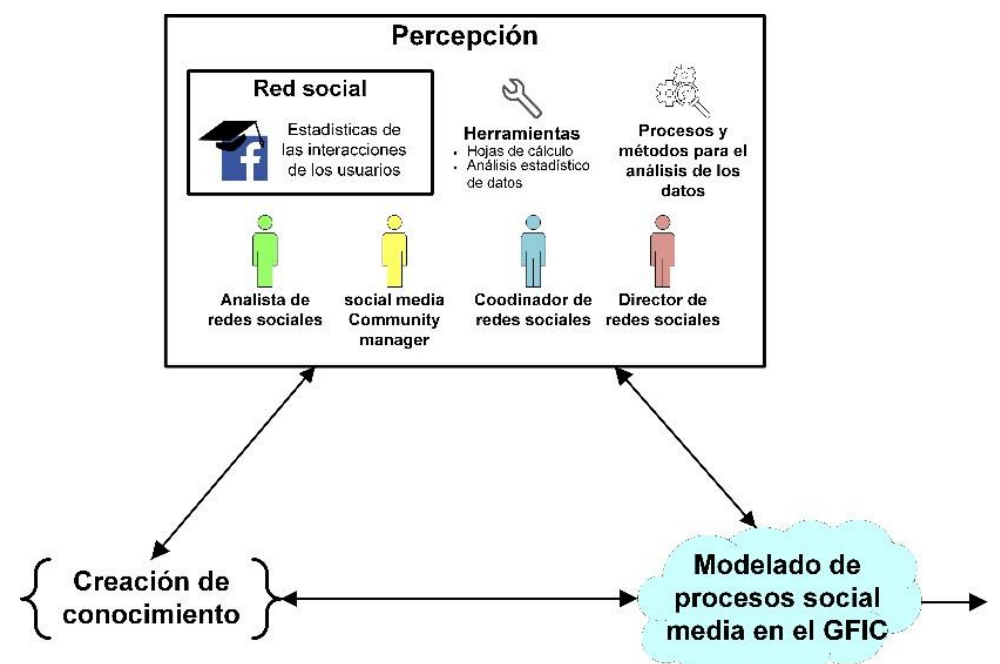

Figura 5. Modelado global del ciclo de conocimiento para procesos de social media. Adaptada de: FloresRios (2016)

\section{Fase 3. Análisis del flujo de conocimiento}

Partiendo del modelo jerárquico Datos-Información-Conocimiento (DIC) (Arias \& Aristizábal, 2011; Davenport \& Prusak, 1998), se planteó una jerarquía inversa (CID) argumentando que el conocimiento se articula en un contexto conceptual, que permite verbalizar el conocimiento para generar información (Tuomi, 1999), que puede ser estructurada en datos independientes. La Figura 6 representa el flujo cíclico y desglosa la distinción entre DIC detectados en los procesos de social media dentro del estudio de caso. El modelo DIC presentó una visión de los datos extraídos desde el grupo GFIC de Facebook, hasta convertirse en información ordenada para representar conocimiento en el análisis. De esta forma, el modelo CID, brindó la posibilidad de articular, verbalizar y estructurar el conocimiento desde los procesos de social media, para desglosarlo en información, que posteriormente se asocia a datos individuales, como variables de interés para estudiar. La representación de los datos con el modelo DIC-CID se asocia al proceso de preparar datos de social media, para cumplir con las actividades de limpiar, reducir e imputar, dar formato y transformar los datos.

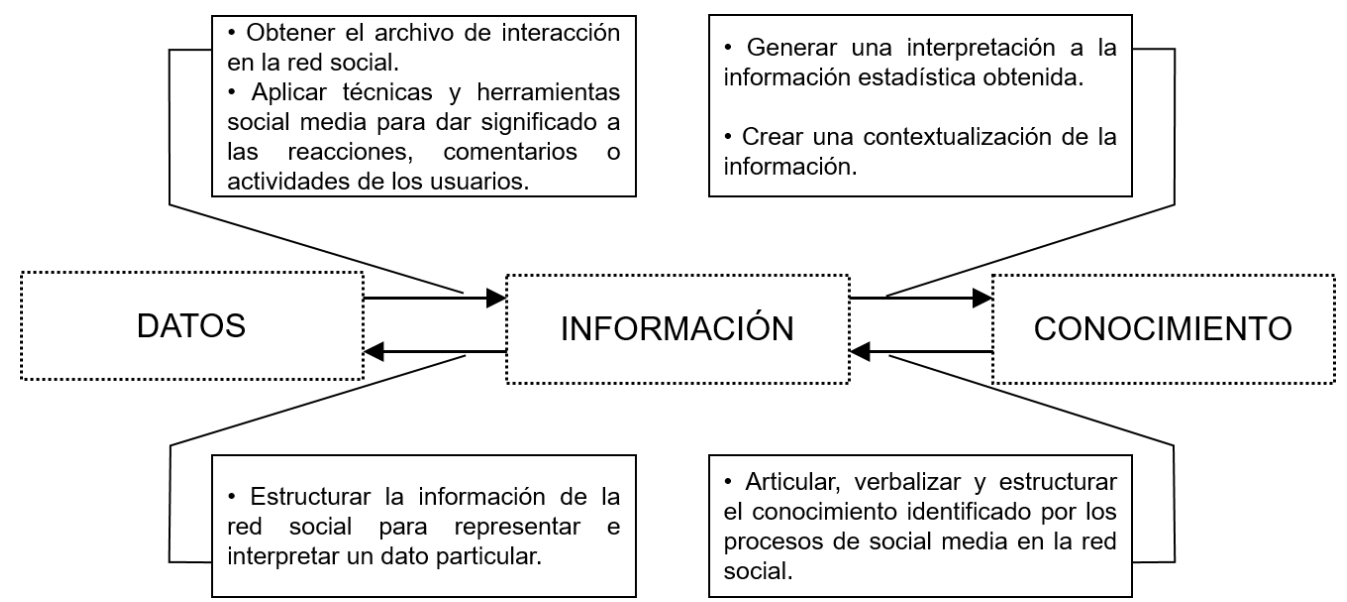

Figura 6. Modelo cíclico Datos-Información-Conocimiento. Elaboración propia

El resultado final de esta fase, permitió elaborar la Figura 7 donde se presentan los flujos de conocimiento identificados en los cuatro procesos de conocimiento de la dimensión epistemológica. De esta forma, se visualiza la tecnología de social media que garantiza el proceso de Socialización, debido a que se genera conocimiento tácito dentro de las redes sociales al intercambiar el conocimiento tácito entre los usuarios. Además, se contribuye a los procesos de Exteriorización e Interiorización el compartir y documentar lecciones aprendidas (habilidades, experiencias, nivel de dominio) de las metodologías relacionadas y las 
técnicas implementadas entre los roles involucrados. El proceso de Interiorización se consideró el más complejo debido a que los roles social media Community Manager y el Analista de redes sociales requieren de la interpretación de los procesos de social media, dando un sentido a la información y el dominio de conocimiento que se genera a partir de las redes sociales y el nivel de conocimiento técnico que se requiere para su análisis. La Combinación es el proceso que involucra la categorización de documentos con los datos utilizados desde las redes sociales, el convertir la información generada por las interacciones en las redes sociales y almacenarla en bases de datos, la generación de gráficas, modelos de análisis o tablas, para lograr dar un sentido más completo a la información. Al aplicar el proceso de Análisis de datos se obtuvo un reporte de interacciones o del comportamiento social virtual.

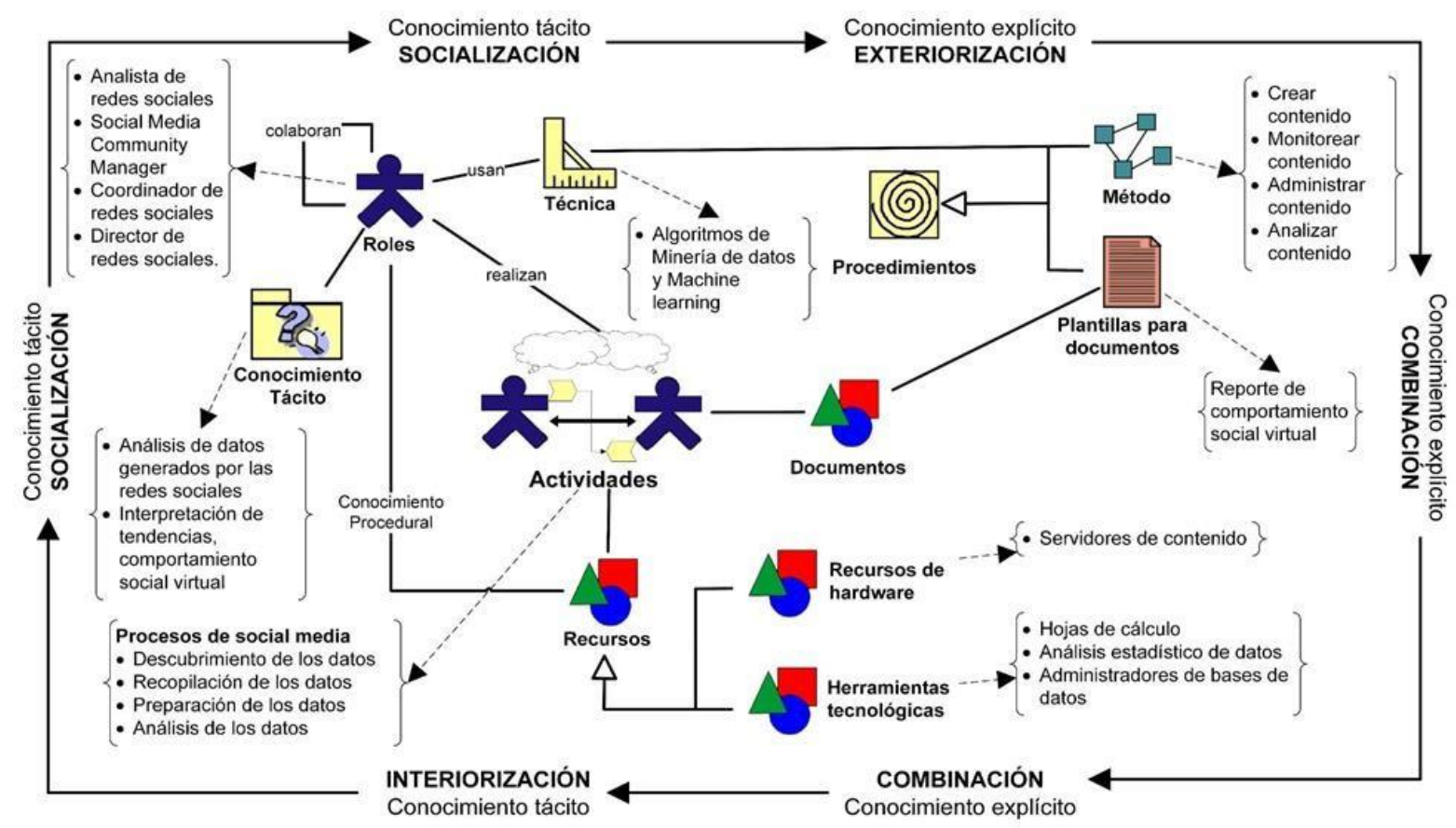

Figura 7. Flujos de conocimiento identificados en procesos de conocimiento de la dimensión epistemológica.

Elaboración propia

Se analizó de manera integral, el conjunto de elementos y relaciones detectados dentro de un contexto de social media (Figura 7) enfocado en el estudio de caso del GFIC. Partiendo del acceso a los datos generados por las actividades y reacciones de los usuarios en las redes sociales (presentados en estadísticas semanales, mensuales o anuales), fue posible una aproximación a la información relevante. El resultado de la fase 3 es el insumo para la toma de decisiones sobre el despliegue de nuevas iniciativas de mejora de los flujos de conocimiento existentes o la creación de procesos de innovación y el análisis de herramientas que apoyan dichos flujos.

\section{Fase 4. Análisis de las herramientas de soporte al flujo de conocimiento}

En la Figura 8 se muestra cada una de las fases de los procesos de social media. Al determinar el dominio de conocimiento y seleccionar el conjunto de datos (GFIC), se realizó una caracterización de la red social y el estudio de caso analizado, se eligieron las herramientas (Excel, SPSS, RStudio) y los métodos (Analizar el contenido). Para la recolección de datos se exportó en un archivo de Excel el periodo de información del semestre 2021-1 del GFIC. La preparación de los datos extraídos se realizó en Excel, SPSS y RStudio. Excel se implementó para limpiar los datos, seleccionando las columnas necesarias para el análisis, RStudio se utilizó para reducir la cantidad de datos al periodo requerido, y se imputaron aquellos que no contenían un valor para el estudio, con SPSS se dio un correcto formato a los datos y en RStudio se transformaron los datos para aplicar un análisis estadístico que diera un acercamiento al comportamiento de los datos. 


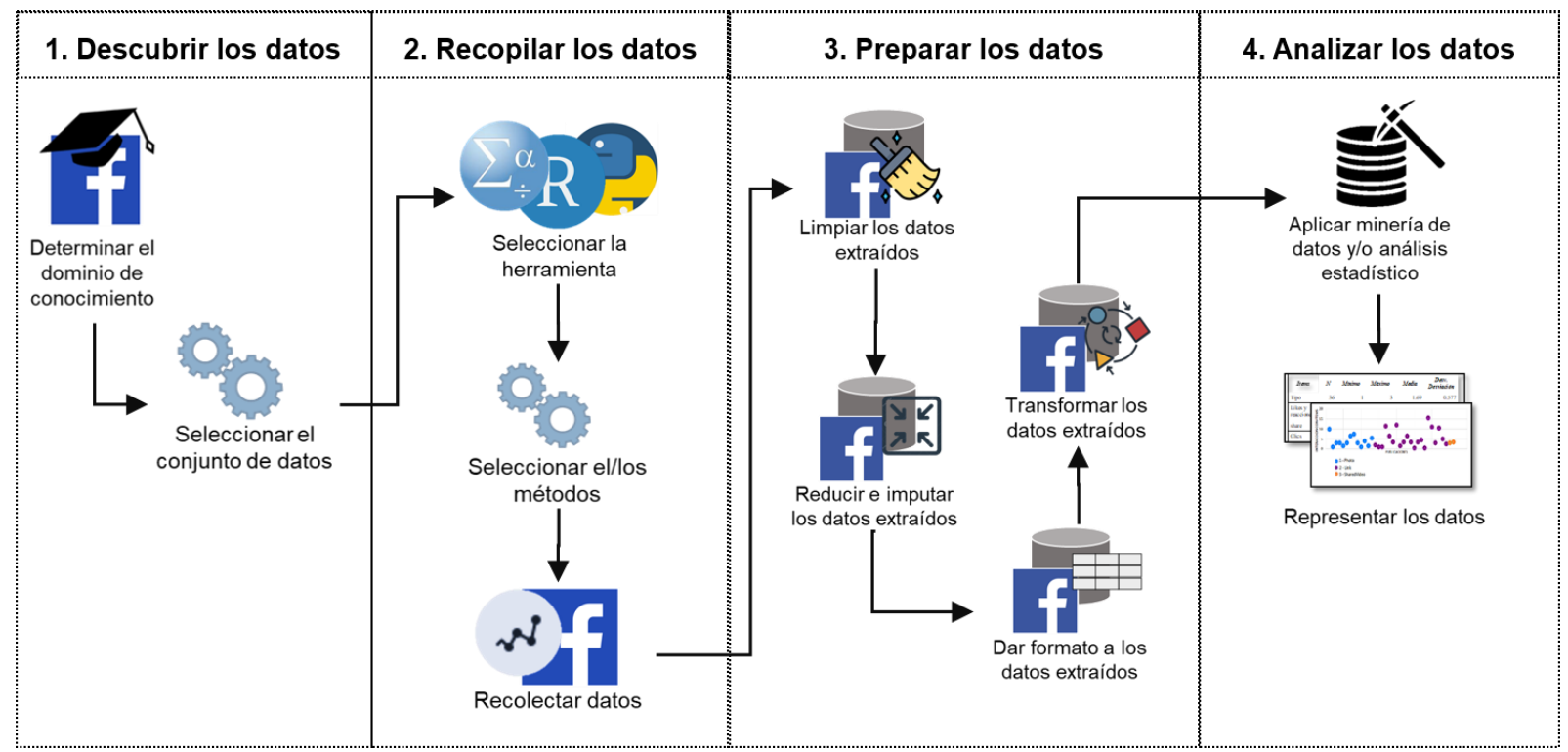

Figura 8. Modelado de procesos de social media. Elaboración propia

El cálculo del engagement $(E)$ para la muestra de datos obtenida del periodo de enero a junio de 2021, se realizó por medio de la fórmula propuesta por Peruta \& Shields (2017).

$$
\mathrm{E}=\frac{\underline{\mathrm{N}}_{\mathrm{r}}+\mathrm{N}_{\underline{\mathrm{s}}}+\mathrm{N}_{\underline{\mathrm{c}}}}{\mathrm{N}_{\mathrm{l}}}
$$

Dónde:

- $\quad \mathrm{N}_{\mathrm{r}}$, Número de reacciones (Me gusta, Me encanta, Me divierte, Me sorprende, Me entristece o Me enoja) en la publicación.

- $\quad \mathrm{N}_{\mathrm{s}}$, número de veces que se ha compartido la página/el grupo de Facebook o la publicación.

- $\quad \mathrm{N}_{\mathrm{c}}$, número de comentarios en la página/el grupo de Facebook o la publicación.

- $\quad \mathrm{N}_{\mathrm{l}}$, número de likes en la página/el grupo de Facebook, o en la publicación. 
La muestra seleccionada presenta el total de datos generados en ese periodo que tengan un valor de interactividad, esto quiere decir que cuentan con publicaciones con comentarios, reacciones y/o han sido compartidas. Un valor de engagement que se encuentre dentro de los intervalos de una página o grupo concurrido y un alto índice de interactividad debe rondar el 0.9 o 1. La Figura 9(a) principalmente presenta un índice de engagement por debajo de la media en páginas y grupos de Facebook concurridos. Presenta poca dispersión y picos en los primeros valores dentro del rango de 0 y 0.1 dentro de la frecuencia. Se identifica una asimetría en el histograma de resultado, lo cual indica que el nivel de compromiso de los miembros del grupo de Facebook analizado es casi nulo. Sin embargo, en la sección de la derecha de la gráfica es posible observar que existen valores atípicos en el rango entre 0.4 y 0.5 , lo cual indica que existe un grupo de miembros del grupo que mantienen una comunicación constante con las publicaciones. Las medidas de tendencia obtenidas del número de miembros activos, exponen una media de 229.58 y una mediana de 155 . La Figura 9(b) presenta asimetría hacia el extremo menor a la cantidad de miembros activos. Por lo tanto, se comprueba que existe una cantidad mínima de miembros activos dentro del grupo. Finalmente, en la Figura 9(c) se busca alguna asociación entre las variables de compromiso y el número de miembros activos, se encontraría una leve correlación positiva (por la dispersión de los puntos) por el movimiento ascendente de los datos hacia la derecha.

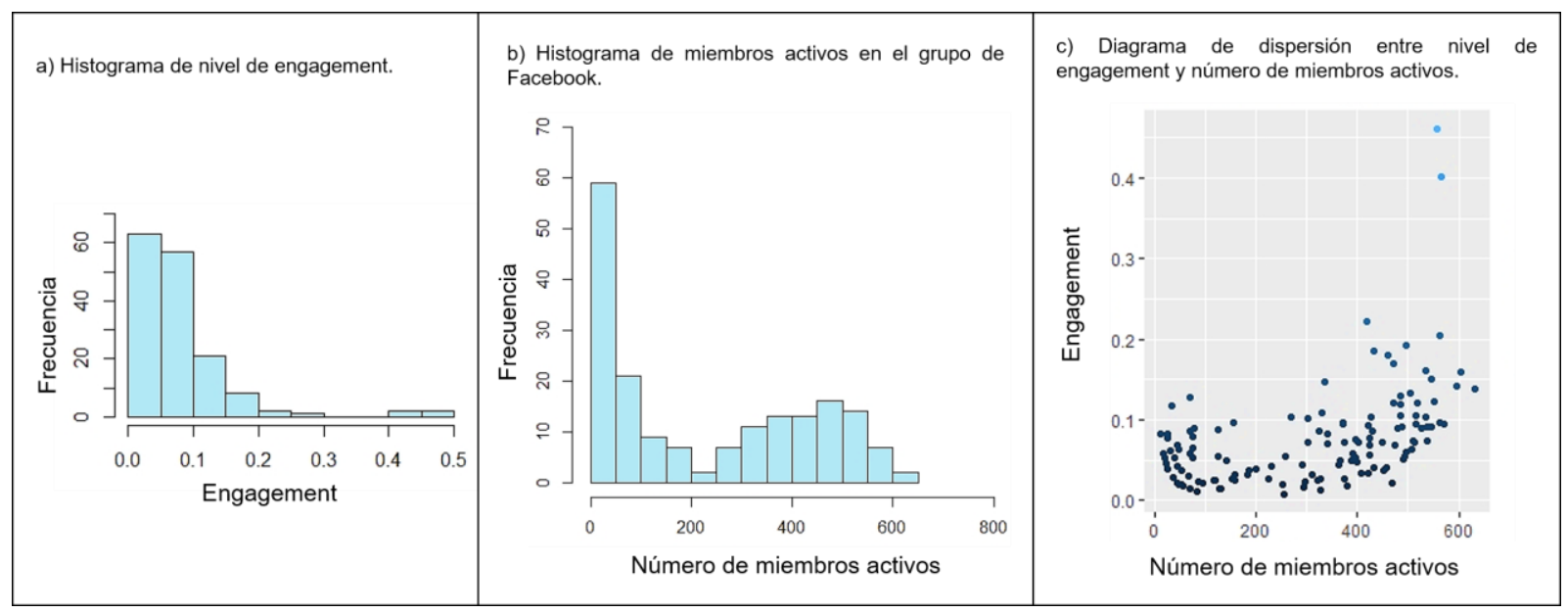

Figura 9. Resultados para el análisis de los datos. Elaboración propia

Del diagrama de dispersión de puntos en la Figura 9(c), se identificaron dos fechas con publicaciones de mayor correlación entre el engagement y el número de miembros activos. Para el día 25 de marzo se obtuvieron un total de seis publicaciones, dos pertenecientes al rol de Social Media Community Manager del GFIC, y cuatro por parte de los miembros. Por otro lado, el día 17 de mayo se realizaron cuatro publicaciones, de las cuales tres fueron por parte de miembros y una por el Social Media Community Manager del GFIC. En ambas fechas, las publicaciones con mayor actividad de reacciones y comentarios corresponden a las realizadas por los Social Media Community Manager. Esto reafirma las características bajo las cuales se estableció el GFIC, como un grupo privado de Facebook con carácter académico informativo, que se utiliza para obtener información relacionada a las actividades de los procesos académicos y anuncios relacionados a sus unidades de aprendizaje del programa educativo. 


\section{CONCLUSIONES}

La aplicación de la GC en el contexto de social media y las redes sociales, como parte de las herramientas para el descubrimiento del conocimiento, permite a las organizaciones identificar, modelar y analizar los flujos de conocimiento de sus activos intangibles. La aplicación de la metodología KoFI permitió obtener la correspondencia y un análisis detallado de los flujos de conocimiento y las herramientas que brindan soporte a los flujos con los cuatro procesos de social media. Así como proponer un flujo para interpretar los contenidos compartidos y las publicaciones asociadas de acuerdo a los diferentes tipos de conocimiento y roles participantes del estudio de caso del GFIC. La fase 1 permitió conocer las fuentes de conocimiento específicas, tanto internas como externas y contener tanto conocimiento tácito como conocimiento explícito. Se identificaron los roles, documentos, procedimientos, sistemas de información y herramientas tecnológicas asociados a los procesos de social media aplicados en una red social bajo un contexto educativo. Se determinó que los roles identificados en la fase 1 , cuentan con conocimiento tácito, relacionado con habilidades, experiencias y conocimientos requeridos y reportados para un adecuado análisis de los datos extraídos desde Facebook. Posteriormente, en la fase 2 se identificaron los tipos de conocimiento involucrados y los flujos de conocimiento existentes en el modelado global del ciclo de conocimiento. Este enfoque de flujos de conocimiento tiene como objetivo brindar un entendimiento de cómo fluye el conocimiento por medio de los cuatro procesos de creación de conocimiento correspondientes a la dimensión epistemológica (Fase 3). Los cuales en la fase 4 se asociaron a los procesos de social media para obtener una retroalimentación de estos en la aplicación de las herramientas y el método seleccionado.

Se concluye que cuanto más especializado sea el grupo de usuarios de la red social, considerando su dominio y nivel de conocimiento tácito, se infiere que habrá mayor exactitud sobre el conocimiento extraído (explícito) con los procesos de social media. Pero, esto sólo funcionará si el contenido de las interacciones de la red social es de calidad. Los resultados de la aplicación de las fases de la metodología KoFI, fueron presentados al social media Community manager del grupo privado GFIC, quien mostró interés en dar seguimiento y monitoreo en el siguiente semestre a cursar.

Como trabajo futuro, se desea trabajar en la interpretación de las estadísticas para mejorar la comunicación entre los miembros y generar nuevo conocimiento explícito. Así mismo, continuar con la identificación de softskills de los roles que están involucrados en cada proceso de social media en apoyo a la selección de técnicas y herramientas. También se recomienda generar un modelo de indicadores que permita analizar los impactos del despliegue de las estrategias de GC en cada proceso de social media.

\section{AGRADECIMIENTOS}

Se agradece al Consejo Nacional de Ciencia y Tecnología (CONACYT) por el apoyo brindado al primer autor con No. de CVU 668484.

\section{REFERENCIAS}

Alavi, M., \& Leidner, D. (2003). Knowledge Management and Knowledge Management Systems: Conceptural Foundations and Research Issues. Journal of Chemical Information and Modeling, 53(9), 16891699.

Alawamleh, H. A. M., \& Murthy, N. (2020). The Influence of Social Media on Organisational Performance and Job Performance. International Journal of Innovation, Creativity and Change, 13(12), 138-156. https://scindeks.ceon.rs/Article.aspx?artid=0409-29532001138K

Ali, A., Bahadur, W., Wang, N., Luqman, A., \& Khan, A. N. (2020). Improving team innovation performance: Role of social media and team knowledge management capabilities. In Technology in Society (61). Elsevier Ltd. https://doi.org/10.1016/j.techsoc.2020.101259 
Arias, J. E., \& Aristizábal, C. A. (2011). El dato, la información, el conocimiento y su productividad en empresas del sector público de Medellín. Semestre Económico, 14(28), 95-109. https://doi.org/10.22395/seec.v14n28a6

Arora, A., Bansal, S., Kandpal, C., Aswani, R., \& Dwivedi, Y. (2019). Measuring social media influencer index- insights from facebook, Twitter and Instagram. Journal of Retailing and Consumer Services, 49(February), 86-101. https://doi.org/10.1016/j.jretconser.2019.03.012

Ballesteros Herencia, C. A. (2018). El índice de engagement en redes sociales, una medición emergente en la Comunicación académica y organizacional. RAZÓN Y PALABRA Primera Revista Electrónica En Iberoamérica Especializada En Comunicación, 22(3_102), 96-124.

Bazarova, N. N., Choi, Y. H., Sosik, V. S., Cosley, D., \& Whitlock, J. (2015). Social sharing of emotions on Facebook: Channel differences, satisfaction, and replies. In Proceedings of the 2015 ACM International Conference on Computer-Supported Cooperative Work and Social Computing (CSCW), 154-164. https://doi.org/10.1145/2675133.2675297

Briciu, V.-A., \& Briciu, A. (2021). Social Media and Organizational Communication. In Encyclopedia of Organizational Knowledge, Administration, and Technology (p. 16). https://doi.org/10.4018/978-1-7998$\underline{3473-1 . \operatorname{ch} 180}$

Cantú Ballesteros, L., Rodríguez Elías, O. M., Sánchez Cuéllar, M. G., Rojas Vásquez, C., Ortega Ruiz, J., Sánchez Córdoba, G. A., \& Becerra Arenas, J. F. (2014). Metodologia de flujo de conocimiento aplicada al proceso de acreditación de programas educativos (segunda fase). Revista Internacional Adminsitración \& Finanzas, 7(1), 31-52.

Chakraborty, A., Dutta, T., Mondal, S., \& Nath, A. (2018). Application of Graph Theory in Social Media. International Journal of Computer Sciences and Engineering, 6(10), 722-729. https://doi.org/10.26438/ijcse/v6i10.722729

Choo, C. (1999). The knowing organization: How organizations use information to construct meaning, create knowledge, and made decisions. Oxford University Press. Oxford, USA.

Claver-Cortes, E., Pertusa-Ortega, E. M., \& Zaragoza-Saez, P. C. (2008). Organizational design and knowledge performance: An empirical study. Intangible Capital, 4(3), 166-190. https://doi.org/10.3926/ic.2008.v4n3.p166-190

Davenport, T. H., \& Prusak, L. (1998). Working knowledge: How organizations manage what they know. Harvard Business Press.

Diaz Ayon, M. (2019). Análisis de la conversión del conocimiento en base al modelo SECI en el área de tecnología de universidades privadas de Lima. In Journal of Chemical Information and Modeling.

Erden, Z., Klang, D., Sydler, R., \& von Krogh, G. (2014). Knowledge-flows and firm performance. Journal of Business Research, 67(1), 2777-2785. https://doi.org/10.1016/j.jbusres.2012.09.001

Flores-Rios, B. L. (2016). Modelo de evolución de la gestión del conocimiento en MiPyMes, de acuerdo con el nivel de madurez en un programa de mejora de procesos de software. In Universidad Autónoma de Baja California. México.

Flores-Rios, B. L., Astorga-Vargas, M. A., Rodríguez-Elias, O. M., Ibarra-Esquer, J. E., \& Andrade, M. del C. (2014). Interpretación de las normas mexicanas para la implantación de procesos de software y evaluación de la capacidad bajo un enfoque de gestión de conocimiento. Revista Facultad de Ingenieria, 71, 85-100.

Flores-Rios, B. L., Pino, F. J., Ibarra-Esquer, J. E., González-Navarro, F. F., \& Rodríguez-Elías, O. M. (2015). Análisis de Flujos de Conocimiento en Proyectos de Mejora de Procesos Software bajo una 
perspectiva multi-enfoque. RISTI - Revista Ibérica de Sistemas y Tecnologías de La Información, 14, 51-66. https://doi.org/10.17013/risti.14.51-66

Giraldo Marín, M. L., Arroyave Cataño, T. E., Galeano Ospino, S., Rojas Mora, J. M., Londoño Montoya, E. M., \& Bedoya Villa, M. A. (2021). Metodología de flujo de conocimiento (primera fase) aplicada la proceso de investigación científica con fines de visibilidad nacional e internacional en la Universidad de Medellín. RISTI - Revista Ibérica de Sistemas e Tecnologias de Informação, 114-127.

Goyal, S. J., \& Goyal, R. (2020). Social Media Analytics - Challenges during the Data Analysis. Artificial \& Computational Intelligence, 1(2). https://acors.org/ijacoi/VOL1_ISSUE2_10.pdf

Gupta, V., \& Gupta, M. (2016). Social Media Mining: A New Framework and Literature Review. International Journal of Business Analytics, 3(1), 58-68. https://doi.org/10.4018/IJBAN.2016010104

Helms, R., Cranefield, J., \& van Reijsen, J. (2017). Social media and knowledge management: A perfect couple. Knowledge Management and Organizational Learning, 3, 1-13. https://doi.org/10.1007/978-3-31945133-6_1

Hu, Y. H., \& Chen, K. (2016). Predicting hotel review helpfulness: The impact of review visibility, and interaction between hotel stars and review ratings. International Journal of Information Management, 36(6), 929-944. https://doi.org/10.1016/j.ijinfomgt.2016.06.003

Kane, G. C. (2017). The evolutionary implications of social media for organizational knowledge management. Information and Organization, 27(1), 37-46. https://doi.org/10.1016/j.infoandorg.2017.01.001

Liu, H., Morstatter, F., Tang, J., \& Zafarani, R. (2016). The good, the bad, and the ugly: uncovering novel research opportunities in social media mining. International Journal of Data Science and Analytics, 1(3-4), 137-143. https://doi.org/10.1007/s41060-016-0023-0

Meng, X., Shi, L., Yao, L., Zhang, Y., \& Cui, L. (2020). Herd Behavior in Social Media: The Role of Facebook Likes, Strength of Ties, and Expertise. Information \& Management, Iii, 124658. https://doi.org/10.1016/j.colsurfa.2020.124658

Mora Garcia, Y. A., \& De la Cruz Chima, M. R. (2018). Diagnostico, desde el modelo SECI de Nonaka y Takeuchi, para proponer estrategias que permitan crear un modelo de gestión del conocimiento en universidades públicas colombianas. Universidad Tecnológica de Bolívar.

Mushtaq, H., Malik, B. H., Shah, S. A., Siddique, U. Bin, Shahzad, M., \& Siddique, I. (2018). Implicit and explicit knowledge mining of Crowdsourced communities: Architectural and technology verdicts. International Journal of Advanced Computer Science and Applications, 9(1), 105-111. https://doi.org/10.14569/IJACSA.2018.090114

Narazaki, R. S., Chaves, M. S., \& Pedron, C. D. (2020). Social media in knowledge management: A holistic knowledge funnel based on a retrospective 10-year study in top-tier journals. Knowledge and Process Management, 27(2), 123-132. https://doi.org/10.1002/kpm.1629

Nisar, T. M., Prabhakar, G., \& Strakova, L. (2018). Social media information benefits, knowledge management and smart organizations. Journal of Business Research, 94(May), 264-272. https://doi.org/10.1016/j.jbusres.2018.05.005

Nonaka, I., \& Takeuchi, H. (1995). The knowledge-creating company: How Japanese companies create the dynamics of innovation. Oxford university press.

Palacios Ospina, M. E. (2017). Técnicas para apoyar la operación combinación de creación del conocimiento, según el modelo SECI. 
Perez-Soltero, A., Barcelo-valenzuela, M., Sanchez-schmitz, G., Martin-rubio, F., Palma-mendez, J. T., \& Vanti, A. A. (2007). A model and methodology to knowledge auditing considering core processes. ICFAI

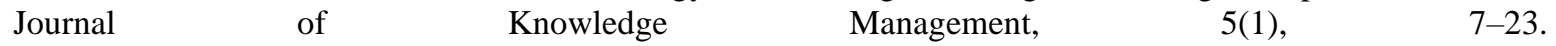
http://citeseerx.ist.psu.edu/viewdoc/download?doi=10.1.1.72.8091\&rep=rep1\&type=pdf

Peruta, A., \& Shields, A. B. (2017). Social media in higher education: understanding how colleges and universities use Facebook. Journal of Marketing for Higher Education, 27(1), 131-143. https://doi.org/10.1080/08841241.2016.1212451

Rodríguez-Elias, O. M. (2007). Metodología para el diseño de sistemas de administración del conocimiento: su aplicación en mantenimiento de software.

Rodriguez-Elias, O. M., \& Martínez, A. I. (2011). Diseño de sistemas y estrategias de gestión del conocimiento: Un enfoque metodológico orientado a procesos y flujos de conocimiento (Spanish Ed). Editorial Académica Española.

Tuomi, I. (1999). Corporate knowledge: Theory and practice of intelligent organizations. Helsinki: Metaxis.

Ureña, R., Chiclana, F., Melançon, G., \& Herrera-Viedma, E. (2019). A social network based approach for consensus achievement in multiperson decision making. Information Fusion, 47(July 2018), $72-87$. https://doi.org/10.1016/j.inffus.2018.07.006

van Rooyen, A. A. (2020). Social media is so easy to share. Accounting Education, 29(4), 356-371. https://doi.org/10.1080/09639284.2020.1779763

Velazquez-Solis, P. E., Flores-Rios, B. L., Ibarra-Esquer, J. E., Astorga-Vargas, M. A., Aguilar Vera, R. A., \& Ramírez-Barreto, Elizabeth López-Morteo, G. (2021). Identificación de áreas de aplicación de arquitecturas de software basadas en modelos, técnicas y herramientas de social media. Revista Ibérica de Sistemas e Tecnologias de Informação (RISTI). 42(06) pp. 12-29. 2021. ISSN: 1646-9895. https://doi.org/10.17013/risti.42.12-29

Vranešević, T., Perić, N., \& Marušić, T. (2019). Perception of Social Media as a Source of Relevant Information. Zagreb International Review of Economics and Business, 22(1), $133-144$. https://doi.org/10.2478/zireb-2019-0016 


\section{NOTAS BIOGRÁFICAS}

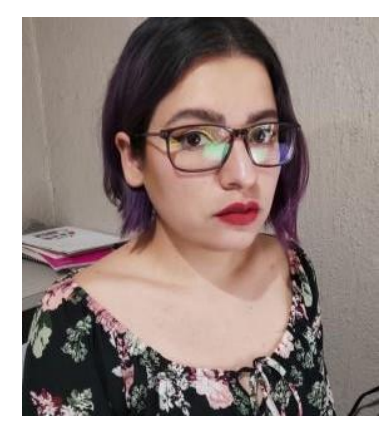

Desarrollo Profesional.

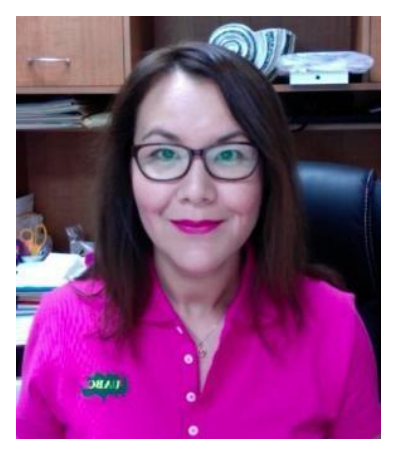

Brenda L. Flores Rios es egresada de Ingeniería en Sistemas Computacionales del Instituto Tecnológico de La Paz, obtuvo su maestría en Ciencias de la Computación en el Centro de Investigación Científica y de Educación Superior de Ensenada (CICESE) y el grado de Doctor en Ciencias por la Universidad Autónoma de Baja California (UABC) en el área de conocimiento de Computación. Es investigadora del área de ingeniería física del Instituto de Ingeniería (II-UABC) e imparte clases en la Facultad de Ingeniería campus Mexicali de la UABC. Pertenece al Cuerpo Académico de Cómputo Científico registrado ante PRODEP-SEP, cuenta con el SNI nivel 1 y es miembro del área de mujeres en la computación de la Academia Mexicana de Computación. Su trabajo de investigación se relaciona con la Ingeniería de Software, Mejora de procesos de Software y Minería de procesos.
Paola E. Velazquez Solis es egresada del programa de Ingeniería en Computación de la Facultad de Ingeniería campus Mexicali de la Universidad Autónoma de Baja California. Cuenta con una Maestría en Ciencias en el área de conocimiento de Computación y actualmente es estudiante becaria CONACYT de Doctorado en Ciencias en el área de conocimiento de Computación. Su trabajo de investigación se centra en temas relacionados con la Ingeniería de Software, Minería de datos, Social media y Analítica de datos. Su experiencia profesional docencia impartiendo clases en universidades privadas como la Universidad del Valle de México, Universidad Autónoma de Durango y Universidad de

\section{(2) $\odot \Theta \odot$}

Esta obra está bajo una licencia de Creative Commons

Reconocimiento-NoComercial-Compartirlgual 2.5 México. 